\title{
Infrastructure Asset Management - A Systems Engineering Perspective
}

\author{
Dr. Fuzhan Nasiri \\ Concordia University, Montreal, QC, Canada
}

\begin{abstract}
Infrastructure asset management has emerged as one of the 21 st century challenges provided an unprecedented pace of urbanization with every increasing demand for infrastructure services against a backdrop of climatic change and more stringent financial resources. The post-COVID economic recovery plans across the globe have emphasized the investments in infrastructure as a means of invigorating the growth. In this sense, the infrastructure sector needs to seize on this opportunity to address the various gaps in management of infrastructures. This lecture provides a whole systems perspective on infrastructure asset management practices. The aim is to systematically characterize the common principles, gaps, and paradigms in infrastructure asset management. This is to advocate an integrated asset management approach that links operational and network-level decisions with the future socio-economic and environmental scenarios to ensure resilience and efficient coordination of infrastructure assets that are exposed to vulnerabilities due to increasing imbalance between levels of service and demand, increasing infrastructure asset complexities and their interdependencies, and the realities of decentralized/distributed management practices.
\end{abstract}

